\title{
AOR
}

Selected Papers of \#AoIR2020: The $21^{\text {st }}$ Annual Conference of the Association of Internet Researchers Virtual Event / 27-31 October 2020

\section{FOLLOW THE MONEY: A LARGE-SCALE INVESTIGATION OF MONETIZATION AND OPTIMIZATION ON YOUTUBE}

\author{
Òscar Coromina \\ Universitat Autònoma de Barcelona \\ Ariadna Matamoros-Fernández \\ Queensland University of Technology \\ Bernhard Rieder \\ University of Amsterdam
}

\section{Introduction}

Since its launch as a video sharing website in 2005, YouTube has become a dominant actor in the global media system, with a high level of social and cultural impact, reaching an audience of almost two billion active users per month (Gilbert, 2018). YouTube also plays the role of a platform in the economic sense, operating at the center of a multi-sided market (Rochet \& Tirole, 2006) and capturing surplus from the intermediation between content creators, audiences, and advertisers. YouTube's business model - which generated \$15B of revenues in 2019 (Statt, 2020) - relies on millions of channels uploading videos, pursuing views and subscribers, and increasingly professionalizing their production methods to become viable businesses themselves. But since the platform first invited content creators to become YouTube "partners" in 2007, allowing them to obtain a share of revenues from advertising, the rules and mechanisms for monetizing videos have changed often and sometimes abruptly, accelerating the search for other income streams.

This paper draws on a critical media industries perspective (Havens et al., 2009) to investigate the relationship between YouTube's evolving platform strategies on the one side and content creators' tactical adaptations on the other. Since the early days of "entrepreneurial vloggers" (Burgess \& Green, 2018, p. 36), the monetisation features and programs the platform offers have evolved significantly, including recent additions such as double pre-roll video advertising, "join" and "clap" features, and the possibility to sell merchandising directly from the interface. The first part of our contribution will reconstruct these developments, but also discuss the increasingly strained relationships between platform, advertisers, and content creators, which first culminated in 2017 , when advertisers pulled out after discovering that their ads were running on videos Suggested Citation (APA): Coromina, O., Matamoros-Fernández, A., Rieder , B. (2020, October). Follow the Money: A Large-Scale Investigation of Monetization and Optimization on Youtube. Paper presented at AolR 2020: The 21 $1^{\text {th }}$ Annual Conference of the Association of Internet Researchers. Virtual Event: AoIR. Retrieved from http://spir.aoir.org. 
promoting terrorism and hate speech (Fontaine et al., 2018), leading to waves of content demonetization. In a situation where advertisement rules are unstable and opaque, creators have turned to product placement, sponsorships, affiliate programs, and crowdsourcing as means to generate income. Patreon has played a particularly important role, since channels dealing with sensitive topics such as politics or sexuality have mostly been excluded from direct monetization altogether. And beyond monetization lurks the broader question of how creators optimize their content and referencing strategies to "make it" on a highly competitive platform that uses dynamic algorithms to modulate visibility (cf. Bishop, 2019).

Establishing an understanding and timeline of these developments provides context and feeds directly into a representative, large-scale empirical analysis of the "microlevel industrial practices" (Havens et al., 2009, p. 235) that content creators employ to generate visibility and revenue on YouTube.

\section{Studying Monetization and Optimization on a Large Scale}

Although recent studies (e.g. Bärtl, 2018) have made important steps toward overall characterizations of YouTube, much work remains to be done. This research draws on a larger project implementing an exhaustive breath-first crawl following subscriptions and "featured channel" connections to map a very large portion of the platform. Collecting data from YouTube's Web-API between November 26, 2019 and January 8, 2020 we created an exhaustive sample of over 36 million channels (Rieder et al., 2020). In this paper, however, we focus on the YouTube "elite" - channels that have already succeeded in finding a significant audience - and thus limit ourselves to the 153.770 channels with more than 100,000 subscribers, as well as the 138.340 .337 videos they posted during their lifespan. For both channels and videos, we were able to collect rich metadata, which allow for a wide variety of analysis.

While the YouTube API provides no means to investigate monetization through advertising empirically, video descriptions contain different traces (e.g. URLs) for alternative forms of income generation. We are thus able to track not only the appearance and spread of crowdfunding platforms like Patreon, but also of affiliate links, merchandise stores, or e-commerce websites like Etsy that focus on low-volume sellers. We pay particular attention to the question of how the progressive exclusion of videos, channels, and whole topic categories (e.g. demonetization of "made for kids" content) has affected the uptake of alternative monetization strategies and to the broader question how these strategies are distributed over algorithmically assigned channel categories (figure 1), which play an important role in how YouTube governs the placing of advertising. 


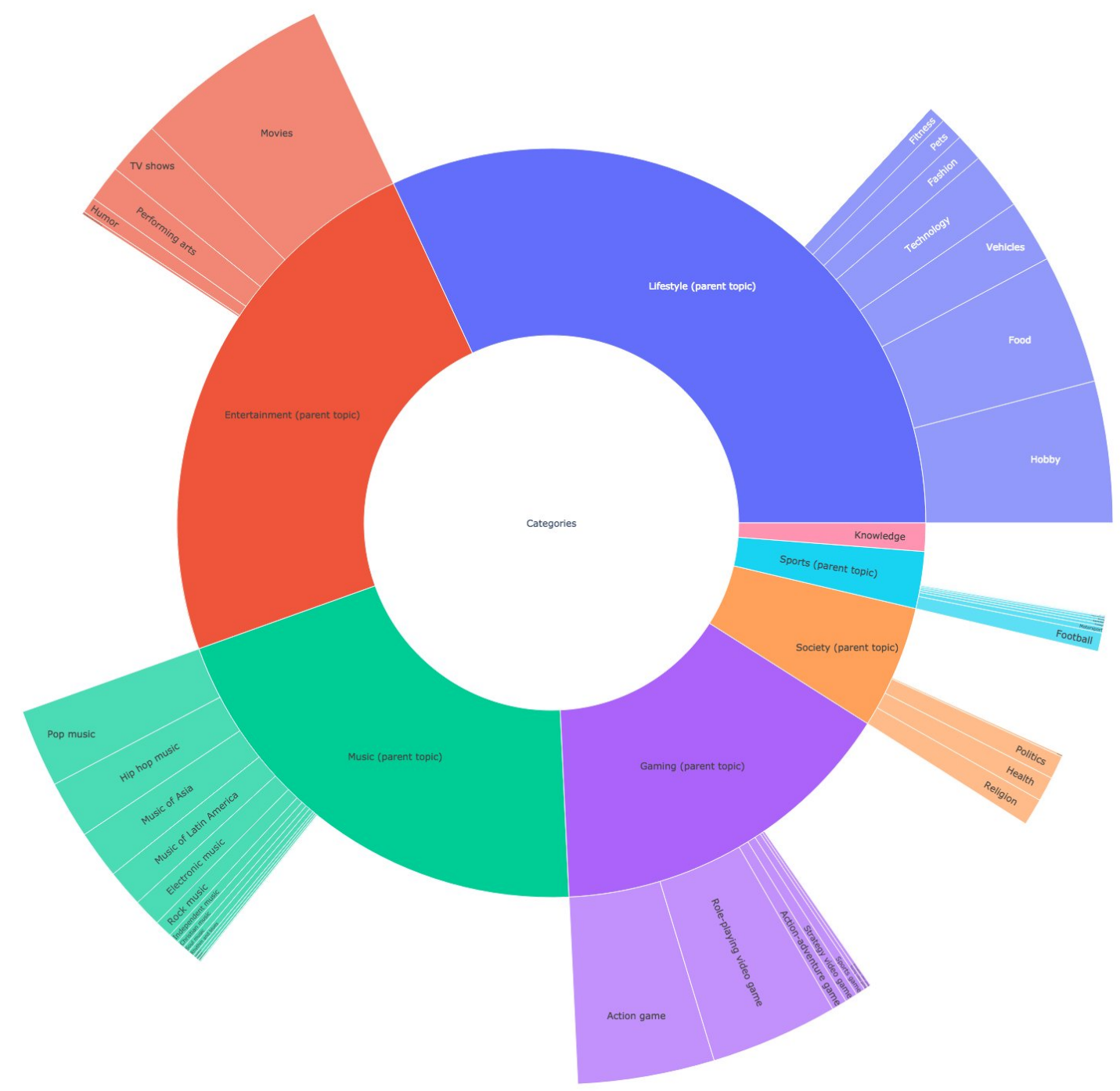

Figure 1. The distribution of 153.770 "elite" channels over YouTube's category grid

Furthermore, we analyze broader "optimization" techniques. This includes the evolution of both video length, one of the parameters that is said to have a strong effect on advertisement revenue, and video posting frequency, another key ingredient in a channel's success. But it also includes practices that seek to enhance algorithmic visibility, such as metadata and category optimization, keyword stuffing, or title phrasing. Controversy is another often-highlighted aspect of YouTube culture that can serve as a means to generate attention. Using like, dislike, and comment ratios of videos as our main indicators, we introduce a "controversiality" metric for channels that allows us to trace discord over topics and sub-communities (i.e. dense clusters in the channel network) and to connect it to the evolution of viewer numbers over time. We are thus able to both investigate controversiality as a strategy and measure its prevalence in the different niches of platform.

Taken together, these elements provide a broad picture of "industrialization" on YouTube, that is, of the ways creators seek to develop their channels into media businesses. 


\section{Conclusion}

While this contribution cannot replace more qualitative, in-depth research into particular channels or channel groups, we hope to provide a representative picture of YouTube's elite channels and their quest for visibility and success from their beginnings up to early 2020. Our method sheds light on YouTube's economic ecology and opens the door to the possibility to classify YouTube channels according to their model of monetization and to analyze relationships between monetization, optimization, and topicality.

\section{References}

Bärtl, M. (2018). YouTube channels, uploads and views: A statistical analysis of the past 10 years. Convergence, 24(1), 16-32.

Bishop, S. (2019). Managing visibility on YouTube through algorithmic gossip. New Media \& Society, 21(11-12), 2589-2606.

Burgess, J., \& Green, J., (2018). YouTube: Online video and participatory culture. 2nd Edition. Cambridge: Polity Press.

Fontaine, G., Grece, C., \& Jimenez Pumares, M. (2018). Online video sharing:

Offerings, audiences, economic aspects. Strasbourg: European Audiovisual Observatory.

Gilbert, B., (2018, May 4). YouTube now has over 1.8 billion users every month, within spitting distance of Facebook's 2 billion. Business Insider.

https://www.businessinsider.com/youtube-user-statistics-2018-5

Havens, T., Lotz, A. D., \& Tinic, S. (2009). Critical media industry studies: A research approach. Communication, Culture and Critique, 2(2), 234-253.

Rieder, B., Coromina, O., \& Matamoros-Fernandez, A. (2020). Mapping YouTube: A quantitative exploration of a platformed media system. First Monday, 25(8).

Rochet, J-C., \& Tirole, J. (2006). Two-sided markets: A progress report. The RAND Journal of Economics, 37(3), 645-667.

Statt, N., (2020, February 4). YouTube is a \$15 billion-a-year business, Google reveals for the first time. The Verge. https://www.theverge.com/2020/2/3/21121207/youtubegoogle-alphabet-earnings-revenue-first-time-reveal-q4-2019 